\title{
Des enseignants en milieu minoritaire francophone améliorent leur enseignement pour la réussite en littératie
}

\author{
ANDRÉ C. MOREAU, BRIGITTE STANKÉ, JOANNE PHARAND, LIZANNE \\ LAFONTAINE \\ Université du Québec en Outaouais
}

\begin{abstract}
Résumé
Cet article vise à comprendre les liens entre l'implantation de dispositifs pédagogiques liés à l'observation et l'analyse des données d'apprentissage réalisée en communauté d'apprentissage professionnelle (CAP) et la progression des apprentissages en littératie d'élèves de huit écoles en milieu minoritaire francophone. Le protocole de recherche de type longitudinal sur une période de trois ans documente cette progression en lecture et en écriture des élèves qui réussissent et ceux qui sont à risque. L'analyse des résultats montre, d'une part, que les élèves à risque et non à risque obtiennent des progrès significatifs dans les rendements en lecture et, d'autre part, qu'un lien étroit existe entre cette progression des apprentissages et le travail en CAP associé aux meilleures pratiques en enseignement de la littératie.
\end{abstract}

\section{Abstract}

This article is aiming at understanding the links between teaching devices of learning observations and data analysis conducted in a professional learning community (PLC) on the progression of learning the literacy of students from eight francophone schools in a minority context. The research protocol of longitudinal type has over a period of three years documents the evolution of learning to read and write of at risk and not at risk students. The analysis of the results in this study demonstrates that at risk and not at risk students obtains significant results in reading achievements and that there is an important link between the progression of learning and work as a PLC combined with a approach focusing on ideal practices in teaching of literacy.

Des enseignants en milieu minoritaire francophone

améliorent leur enseignement pour la réussite en littératie ${ }^{I}$

Cet article présente les résultats d'une recherche de type longitudinal sur le développement de compétences en littératie où enseignants et chercheurs travaillent ensemble à améliorer, dans des classes de premier cycle du primaire, les pratiques pédagogiques inspirées des écrits scientifiques (Leclerc et Moreau, 2010). Ces pratiques pédagogiques renouvelées concernent la démarche d'observation des apprentissages et la régulation quant aux meilleures interventions à adopter par les enseignants pour répondre aux besoins d'apprentissage des élèves. Le personnel enseignant de huit écoles en milieu minoritaire francophone participant à cette recherche a également choisi de fonctionner en communauté d'apprentissage

\footnotetext{
${ }^{1}$ Cette recherche a été financée par le Conseil scolaire de district du Centre-Sud de Toronto, Ontario (recherche en littératie 2007-2010) et par l'Équipe de recherche en littératie et inclusion (ÉRLI) de l'Université du Québec en Outaouais. Cette recherche et les résultats présentés dans ce texte s'appuient sur les travaux ayant été documentés dans le rapport de recherche publié par Leclerc et Moreau (2010) et les résultats des données recueillies sur la période 2007-2010 qui n'ont jamais fait l'objet d'une publication.
} 
professionnelle $\left(\mathrm{CAP}^{2}\right)$. L'hypothèse est de croire que l'implantation de pratiques efficaces peaufinées lors d'activités de régulation en équipe collaborative (CAP) a un effet sur les résultats d'apprentissage en lecture d'élèves âgés de six à neuf ans de milieux francophones canadiens (Dufour \& Eaker, 2004; Eaker, Dufour \& Dufour, 2004; Fullan, 2001). Cette démarche de recherche vise à préciser l'apport de ces pratiques pédagogiques renouvelées sur la trajectoire d'apprentissage en lecture d'élèves à risque et d'élèves non à risque.

Les sections suivantes précisent la problématique et le cadre théorique qui soutiennent la démarche de recherche. Enfin, les résultats soulèvent une réflexion sur les dimensions ciblées, l'observation et la régulation des pratiques d'enseignement différencié qui alimentent la discussion sur les pistes de recherche.

\section{La problématique liée à l'enseignement et à}

l'apprentissage de la lecture en milieu minoritaire francophone

Au Canada, l'état de l'apprentissage en littératie fait l'objet d'une veille par les gouvernements à l'échelle fédérale, provinciale ou territoriale; le rapport du Conseil canadien sur l'apprentissage $\left(\mathrm{CCA}^{3}, 2007\right)$ rappelle, entre autres, que quatre adultes sur 10 possèdent des compétences en littératie de base «si faibles qu'elles limitent leurs perspectives économiques » (p.4). Pour la société, la littératie constitue un facteur d'épanouissement des personnes, de développement économique, de cohésion sociale et de vie démocratique. En milieu minoritaire francophone canadien, les compétences en littératie des personnes demeurent un enjeu déterminant pour la réussite scolaire des élèves (Conseil supérieur de l'éducation [CSÉ], 2010). Pour certains chercheurs, il en va de la survie de la culture et de la langue française en milieu minoritaire canadien (Landry, 2003). Ce défi de la réussite scolaire de tous les élèves en milieu minoritaire se combine au contexte d'écoles inclusives où les interventions éducatives tiennent compte des besoins diversifiés des élèves, ceux qui réussissent et ceux à risque (Rousseau, 2010).

Dans ces écoles inclusives en milieu minoritaire accueillant tous les élèves, le problème de l'enseignement à des groupes hétérogènes est une réalité importante à considérer, sachant que les compétences en littératie représentent des capacités essentielles à la réussite scolaire. Or, dans ce milieu où on retrouve un grand nombre d'élèves dont la langue première en milieu familial est différente de la langue d'enseignement, il s'avère difficile en début de scolarité de s'approprier les compétences du langage écrit (lecture et écriture) afin de lire pour apprendre et d'apprendre pour le reste de sa vie (CCA, 2011). En plus des problèmes rencontrés par les élèves à risque ou ceux ayant des difficultés d'apprentissage s'ajoute, chez les élèves en milieu minoritaire, une connaissance insuffisante de la langue d'enseignement, le français. Cette situation s'observe par une absence de l'usage de la langue française ou par un faible niveau de maitrise de l'oral manifesté, entre autres, par un manque de vocabulaire et par une maitrise incomplète de la syntaxe du français. Ces problèmes à l'oral ont des incidences sur les connaissances construites à l'écrit qui se constatent par un faible niveau d'intégration des processus spécifiques

\footnotetext{
${ }^{2}$ La CAP réfère au travail en équipe collaborative (interactions/discussions) du personnel enseignant d'une école fonctionnant d'une façon interdépendante, dont chaque membre est mutuellement responsable et redevable de l'amélioration des résultats et de la réussite des élèves.

${ }^{3}$ Le Conseil canadien sur l'apprentissage (CCA) était un organisme indépendant à but non lucratif financé en vertu d'une entente avec Ressources humaines et Développement social Canada. Il avait pour mission de favoriser et de soutenir les décisions fondées sur des éléments objectifs au sujet de l'apprentissage à tous les stades de la vie, depuis la petite enfance jusqu'au troisième âge. Le CCA a cessé d'être financé par le gouvernement canadien en 2011.
} 
d'apprentissage en lecture comme la connaissance des lettres, l'identification de mots ou l'accès lexical des mots, des processus d'intégration propres au traitement sémantique pragmatique liés à la difficulté de compréhension de phrases ou de textes (Montésinos-Gelet, 2009). Ces problèmes font écho au défi, sinon à la difficulté, des enseignants en milieu minoritaire francophone de traduire les résultats de recherches en des pratiques efficaces d'enseignement de la littératie dans leur classe.

Or, notre travail en partenariat avec des écoles inclusives en milieu minoritaire a permis-d'implanter un mode de fonctionnement en CAP et d'optimiser les pratiques d'enseignement efficace en littératie (lecture-écriture) qui s'appuient sur des résultats probants de recherches (Crahay, 2000; McCardle, Chhabra et Kapinus, 2008; National Reading Panel, 2000). À cet égard, le ministère de l'Éducation de l'Ontario (Secrétariat de la Littératie et de la Numératie, 2007) reconnait un rôle de premier plan à la collecte de données d'apprentissage ${ }^{4}$ lors du processus d'enseignement et d'apprentissage : informer l'élève de la progression de ses apprentissages et, pour le personnel enseignant, susciter le questionnement afin de prendre des décisions éclairées quant aux meilleures interventions. L'analyse des écrits scientifiques sur le processus d'enseignement et d'apprentissage renseigne sur : a) les premières habiletés essentielles d'apprentissage menant à un niveau de lecture fonctionnelle; b) les outils ou méthodes reconnues pour observer les premières acquisitions en lecture; c) les liens entre les observations et les actions pédagogiques réfléchies mises en place; et d) les apports des recherches de type longitudinal.

\section{Les habiletés essentielles au développement des compétences en littératie}

La documentation scientifique nous apprend que la littératie peut être comprise comme une compétence générale qui permet à toute personne d'avoir accès au monde extérieur, d'interagir, de communiquer, d'apprendre et de socialiser (Grenier, Jonse, Strucker, Murray, Gervais \& Brink, 2008; Moreau, Hébert, Lépine \& Ruel, 2013). La « compétence en littératie » désigne les capacités d'utiliser le langage et les images de formes riches et variées pour voir, écouter, parler, lire, écrire, comprendre, communiquer et penser de façon critique afin d'atteindre un but et de développer ses connaissances en classe, à l'école, à la maison ou dans la communauté (ministère de l'Éducation de l'Ontario [MEO], 2005). Plusieurs recherches soutiennent que l'acquisition des compétences en littératie amène l'élève à mieux comprendre les liens existants entre la lecture, l'écriture et la communication orale. Ce corollaire implique que le développement de l'une des compétences langagières (oral [réceptif-expressif], lecture et écriture) a des répercussions sur les deux autres ou, encore, que les trois types d'activités ont des processus communs dans l'apprentissage d'une langue (Fitzgerald \& Shanahan, 2000; Mallozzi \& Malloy, 2007; Reuter, 1998). Il existe des liens forts entre les performances des élèves dans des activités à l'oral, par exemple, sur les phonèmes et les progrès dans l'apprentissage de la lecture (Courcy, 2000; Fitzgerald \& Shanahan, 2000; Fountas \& Pinnell, 2001; Jaffré, 2003); ces résultats sont de puissants indicateurs de l'apprentissage de la lecture (Giasson, 2011).

\footnotetext{
${ }^{4}$ Le terme « données d'apprentissage » désigne les éléments observés chez un élève lors de ses activités d'apprentissage : comportement et verbalisation pouvant décrire un élève dans ses essais d'apprentissage. Ces éléments sont des représentations d'information sous forme conventionnelle (données qualitatives ou quantitatives) pour en faciliter son traitement. Ces données s'inscrivent dans une démarche de collecte réalisée en cours d'activités d'apprentissage (Legendre, 2005, p. 88 et p. 437). Ces observations recueillies à l'aide d'instruments divers permettent de décrire les interactions et l'intégration d'objets d'apprentissage chez l'élève (Table ronde des experts, 2005, p. 21).
} 
Ainsi, l'appropriation des concepts reliés à l'écrit, la conscience phonologique, l'acquisition du système graphophonémique et l'étude de mots sont des composantes essentielles menant à l'acquisition de la compétence à lire (Bara, Gentaz \& Colé, 2004; Cormier, 2006; Ecalle \& Magnan, 2002, 2010; Foulin, 2007; Giasson, 2011). C'est pourquoi les activités en langage écrit doivent occuper une place centrale pour que l'élève puisse arriver à utiliser conjointement les trois systèmes d'indices de lecture, soit graphophonémique, sémantique et syntaxique (Giasson, 2011). Le développement de ces apprentissages, loin d'être homogène, donne lieu à des trajectoires différentes d'un élève à l'autre. En fait, les connaissances (processus) menant au décodage et à l'identification des mots et de phrases sont importantes, mais insuffisantes pour mener à la compréhension (Blanc, 2009).

En fait, les recherches sur les élèves en difficulté de lecture ont permis de préciser qu'un bon lecteur en identification de mots n'était pas toujours bon en compréhension (Blanc, 2009). Il existe deux grandes composantes dans l'apprentissage de la lecture : une première liée aux connaissances et aux processus de base de l'identification de mots et une seconde propre aux processus cognitifs de haut niveau liés à la compréhension. Ces deux composantes distinctes se développent entre l'âge de trois et cinq ans (Blanc, 2009; Makdissi \& Boisclair, 2006). Les compétences premières en compréhension s'acquièrent d'abord à l'oral, puis à l'écrit de façon implicite en situation d'interaction enfant-adulte et enfant-enfant et se poursuivent de façon explicite lors de la scolarisation formelle (Blanc, 2009; Ecalle \& Magnan, 2010).

En outre, les premières habiletés de compréhension, comme attribuer une représentation à une image, à un titre ou à un texte lu par un adulte, ou même une représentation d'histoire télévisée, ou simplement se raconter une histoire vue ou écoutée, sont observées chez l'enfant avant qu'il n'apprenne à lire. Ces mêmes habiletés de haut niveau de la pensée se manifesteront plus tard en contexte de lecture de textes lus par l'adulte à l'élève ou par l'élève (Blanc \& Brouillet, 2003; Van den Broek et al, 2005). Somme toute, la compréhension en langage écrit est fonction de : a) l'accumulation d'expériences riches en littératie qui permettent à l'enfant d'enrichir ses connaissances générales sur le monde; et b) l'appropriation de stratégies efficaces d'acquisition d'informations propres aux compétences de haut niveau comme se rappeler, inférer et interpréter.

\section{L'observation de la compétence à lire comme nouvelles pratiques d'enseignement}

Clay (2003) s'est intéressée aux premiers apprentissages essentiels du savoir lire-écrire où la lecture de livres jeunesse est au centre du développement des compétences en littératie. Ses travaux l'ont menée à valider une démarche d'observation des comportements de jeunes lecteurs. Cette démarche regroupe différents outils, dont la Fiche d'observation individualisée (FOI), l'identification des lettres, les concepts reliés à l'écrit et le Test de mots (Clay, 2003; Nelley \& Smith, 2003). L'outil le Sondage d'observation constitue un atout majeur lors de l'analyse et $\mathrm{du}$ questionnement efficace relatif aux apprentissages en lecture. Le matériel privilégié pour les situations d'observation est la lecture de récits. Les livres d'histoires sont intéressants en raison des situations réelles qu'ils évoquent et pour leur facilité à les comprendre et à se les rappeler. Ils ont comme particularité de mobiliser les mêmes connaissances que celles utilisées pour comprendre les événements qui se présentent dans la vie quotidienne et dans le monde réel (Blanc, 2009). 
Dans la mesure où les progrès des élèves sont évalués et examinés fréquemment, les observations des niveaux de lecture et des comportements du lecteur fournissent un indice du progrès en lecture. Cette progression s'exprime, entre autres, par le passage d'un niveau de lecture à un autre : par une lecture à haute voix, l'élève doit démontrer sa capacité à lire d'une façon satisfaisante des textes d'un niveau spécifique représentant un défi sans pour autant être en situation d'échec avant de passer à un autre niveau de texte. Par ses expériences, l'élève-lecteur développe au fur et à mesure de nouvelles stratégies pour faire face à l'augmentation du niveau de difficulté de texte (Clay, 2003). Pour qu'il y ait apprentissage, il est indispensable de s'assurer que le niveau des textes proposés à l'élève représente un défi qui le stimulera à vouloir lire davantage. Ainsi, pour donner accès à la littérature jeunesse, le niveau de texte de tous livres des classes ou bibliothèques des écoles participant à la recherche a été identifié. Ce niveau de difficulté de lecture doit être bien ciblé, car s'il constitue un obstacle trop difficile à surmonter, l'élève risque de se décourager dans son apprentissage. Ce passage d'un niveau à un autre est un facteur de progrès. L'analyse de ces données par le personnel enseignant incite à dégager des interventions pédagogiques concrètes pour que tous les élèves puissent progresser; c'est ce que nous appelons ici la régulation de l'enseignement et de l'apprentissage.

\section{La régulation en enseignement comme processus de changement}

Réaliser des observations, avec des outils qui sont reconnus, exige du temps et de la rigueur de la part des enseignants. Cet investissement porte ses fruits dans la mesure où les enseignants s'accordent des moments de qualité pour faire une analyse de leurs données, comme lorsqu'ils se rencontrent en équipe collaborative (CAP) pour réfléchir aux actions pédagogiques à mettre en place pour soutenir l'apprentissage des élèves, c'est-à-dire lors d'activités de régulation.

Comme représentation synthétique du processus d'enseignement-apprentissage, la régulation se développe au cours d'activités pédagogiques qui mènent à l'émergence d'actions fines et ajustées de prises de décision, liées aux observations et propres à la planification et au pilotage de l'intervention de l'enseignant (ministère de l'Éducation du Québec [MEQ], 2001). Elle s'opère par la prise de conscience des enchaînements procéduraux d'une démarche d'enseignement-apprentissage, où s'observe une séquence d'actions de prises de décision planifiées liées à l'intention ciblée. Pour assurer une régulation effective, l'enseignant doit disposer de renseignements pertinents et fiables sur l'apprentissage, pouvoir les interpréter correctement pour inférer les étapes d'apprentissage subséquentes ou les difficultés à surmonter de la part de l'élève et, ainsi, imaginer les interventions appropriées qu'il pourra piloter efficacement (Perrenoud, 1998).

Ainsi, la régulation passe, d'une part, par des observations de qualité, fiables et pertinentes recueillies par un enseignant formé et instrumenté (Perrenoud, 1998). D'autre part, l'observation doit être cohérente, en continuité et en adéquation au regard des interventions régulatrices à instaurer. Enfin, cette régulation effective rend accessible à l'élève une rétroaction ou, au plus, un étayage par des questions, des renseignements ou des suggestions qui le guident vers une progression optimale de son apprentissage (Perrenoud, 1998). Cette capacité d'analyser les actions pédagogiques et de réguler s'inscrit dans la poursuite de l'appropriation des liens théorie-pratique amorcée lors de la formation initiale en enseignement et, d'une façon plus précise, dans la construction de principes et de règles pédagogiques. En outre, pour y parvenir, l'enseignant peut développer sa capacité de régulation en multipliant les situations où il peut analyser ses actions et les indices de compréhension- 
apprentissage observés chez les élèves. Ces analyses lui permettent de parfaire les règles et les principes pédagogiques qui modulent les prises de décision lors de son enseignement. Il s'agit de passer d'une régulation intuitive qui ignore les raisons de l'efficacité de ses actions à une régulation réfléchie menant à faire des choix d'interventions plus adaptées et plus efficaces (Miffre, 1992). Le travail en équipe collaborative prend ici tout son sens (communauté d'apprentissage professionnelle [CAP]).

Essentiellement, le fonctionnement en équipe collaborative structuré dans une école fonctionnant en CAP assure au personnel des moments de qualité et un contexte de travail d'équipe qui, par des relations d'entraide, permettent l'analyse des données d'apprentissage et des interventions les plus susceptibles de faire progresser tous les élèves dans leurs apprentissages (Dufour \& Eaker, 2004; Fullan, 2001; Leclerc \& Moreau, 2009, 2010; Moreau, Leclerc \& Stanké, 2013). Le fonctionnement en CAP, comme outil de changement, crée une synergie menant les enseignants à créer un solide niveau de concertation, à démontrer une persévérance dans leurs efforts d'améliorer leurs interventions et à assurer une continuité des interventions d'une année à l'autre. Ces rencontres collaboratives nécessitent : a) du temps de qualité pour les réaliser (période de deux heures aux quatre à six semaines); b) une animation structurée (ordre du jour, thème choisi par le groupe d'enseignants, rôles distribués et procès-verbal assurant le suivi); c) une priorité accordée à l'analyse des données d'apprentissage d'élèves éprouvant des difficultés; et d) un leadership partagé, c'està-dire une participation active des enseignants et de la direction aux prises de décision (Moreau, Leclerc \& Stanké, 2013). La mise en place de nouveaux dispositifs pédagogiques favorisant la réussite des apprentissages en littératie comme le fonctionnement en CAP et la régulation des interventions a mené à scruter les écrits scientifiques.

\section{Les recherches longitudinales et les difficultés en lecture}

Partant des écrits scientifiques recensés, plusieurs recherches sur l'enseignement explicite en lecture-écriture auprès d'élèves à risque ou en difficulté concluent à des résultats positifs: enseignement systématique/explicite (Allor, Champlin, Gifford \& Mathes, 2010; Baylis \& Snowling, 2012; Lemons \& Fuchs, 2010; Shanahan \& Lonigan, 2010); et enseignement multisensoriel et systématique (Browder, Ahlgrim-Delzell, Courtade, Gibbs \& Flowers, 2008; Scheffel, Shaw \& Shaw, 2008). À titre d'illustration, la recherche longitudinale expérimentale d'Allor, Mathes, Roberts, Cheatham et Champlin (2010) montre que les élèves ayant des difficultés d'apprentissage peuvent apprendre à lire et à écrire en contexte d'enseignement cohérent, explicite et systématique sur une longue période de temps. Cette étude portait sur 28 élèves âgés de neuf ans en moyenne et ayant des troubles d'apprentissage ou des déficits cognitifs. Seize d'entre eux ont été assignés à la condition expérimentale et les 12 autres élèves ont constitué le groupe contrôle. Ces 12 élèves bénéficiaient d'un enseignement spécialisé typique contrairement aux 16 élèves du groupe expérimental qui ont participé à des séances d'enseignement de 40 à 50 minutes par jour en petits groupes (deux à quatre), et ce, au cours d'une année scolaire et demie (300 séances au total). L'intervention comprenait un enseignement systématique et explicite des concepts de l'écrit, de la conscience syllabique, phonologique et phonémique, de la connaissance des lettres, du décodage et de son automatisation, du vocabulaire et des stratégies de compréhension de lecture (Allor, Mathes, et al, 2010). Les résultats sont significatifs: les élèves du groupe expérimental se distinguent de ceux du groupe contrôle quant à la conscience

\begin{tabular}{lll}
\hline Language and Literacy $\quad$ Volume 16, Issue 2, Special Issue 2014 Page 95
\end{tabular}


phonologique, à la reconnaissance de mots, au vocabulaire et à la compréhension de l'écrit. Ces résultats permettent de préciser quatre facteurs critiques pour une intervention efficace: (a) un degré d'intensité d'enseignement suffisant (certains élèves ayant des difficultés d'apprentissage ou un déficit cognitif ont besoin de trois ans pour maitriser le décodage); (b) un enseignement adapté au niveau d'habiletés de chaque élève, lequel est appuyé par des évaluations régulières, ainsi qu'une intervention régulée et concentrée sur les habiletés non maitrisées; (c) des tâches favorisant l'engagement des élèves dans l'activité selon des objectifs précis; et (d) des activités permettant aux élèves de donner un sens à leur apprentissage, en décodant des phrases puis des textes qui contiennent les mots qu'ils savent lire. Enfin, les auteurs ajoutent que, sans être la principale stratégie de lecture, la reconnaissance de mots lus globalement reste une stratégie à stimuler pour permettre à l'élève de construire un lexique mental qui facilite l'identification rapide de mots.

En outre, la qualité des enseignements explicites est étroitement associée à la précocité, la spécificité et l'intensité de l'intervention, à savoir : (a) l'identification des élèves à risque de difficultés en lecture grâce à un dépistage universel; (b) la détermination des besoins spécifiques des élèves de mêmes capacités jumelées à l'intervention en petits groupes; (c) le matériel quotidien authentique et des stratégies d'intervention explicites et systématiques; (d) les modalités d'intervention spécifique alignées aux activités d'enseignement de la lecture en classe; et (e) l'usage de textes dont le niveau correspond aux besoins des élèves. Ajoutons à ces conditions que les élèves à risque ou en difficultés bénéficient davantage d'un enseignement en petits groupes, car il augmente les possibilités de pratiquer où s'ajoutent les commentaires et la régulation de l'intervention (Burns, Appleton \& Stehouwer, 2005; Ridgeway, Price, Simpson \& Rose, 2012; Vaughn, Denton \& Fletcher, 2010). Enfin, pour certains élèves, la réussite de ce type d'intervention peut demander des modèles plus complexes d'enseignement explicite, y compris une intervention plus intensive (Wanzek \& Vaughn, 2007).

\section{Les objectifs de cette recherche}

Cette recherche vise donc à décrire les trajectoires des apprentissages en littératie d'élèves de la première (six ans) à la fin de la troisième année (neuf ans) du primaire, ainsi qu'à connaître les relations entre la progression des apprentissages et les dispositifs pédagogiques déployés que sont l'observation, la régulation, dont l'implantation de nouvelles pratiques pédagogiques, et le fonctionnement en CAP.

\section{Le protocole et le milieu de recherche}

\section{La méthodologie de la recherche}

Le protocole de recherche est de type longitudinal à mesures répétées sur une période de trois ans de collecte de données d'apprentissage auprès d'élèves $\mathrm{du}$ premier cycle du primaire. Le personnel enseignant de huit écoles (58 enseignants et huit membres à la direction ${ }^{5}$ ) a accepté de collaborer à la recherche (2006-2010). Ces écoles en milieu minoritaire francophone présentent des profils différents. Deux écoles ont des rendements en lecture élevés, trois autres ont des rendements faibles et les trois dernières écoles ont un rendement équivalent à la moyenne du Conseil

\footnotetext{
${ }^{5}$ Nous tenons à remercier les enseignants et membres des directions d'écoles pour avoir contribué à cette recherche.
}

Language and Literacy $\quad$ Volume 16, Issue 2, Special Issue 2014 Page 96


scolaire $^{6}$ au Test provincial. Chez les enseignants, quatre d'entre eux sont de sexe masculin et 54 sont de sexe féminin. Leur moyenne d'âge est de 39,5 ans et leur moyenne d'années d'expérience en enseignement est de 13 ans.

\section{Les participants}

Les élèves participants, âgés de cinq à neuf ans, sont au nombre de 668 (38 groupes-classes), ce qui correspond à $20,87 \%$ de la population du Conseil scolaire. Les variables contrôlées sont l'âge, le sexe, le niveau socio-économique du milieu scolaire (revenu moyen des parents de chaque école), la langue parlée à la maison et les services adaptés. Parmi ces caractéristiques, soulignons que pour la majorité de ces élèves en milieu minoritaire, le français constitue la deuxième langue, l'anglais étant généralement la langue parlée à la maison.

\section{Les mesures}

Les mesures de la progression des apprentissages en lecture sont au nombre de trois: (a) les résultats au Test provincial de l'Office de la qualité et de la responsabilité en éducation (OQRE, 2009, 2010), lequel offre des résultats à grande échelle pour tous les élèves de fin de troisième année, en précisant un niveau de lecture et en identifiant ceux ayant besoin d'un appui (élèves à risque); (b) les données issues de la fiche d'observation individualisée (FOI) (Clay, 2003) et enregistrées dans une base de données fournissant ainsi un indice du niveau de lecture et de la progression en cours d'apprentissage; et (c) les résultats des bulletins scolaires (répertoriés dans la base de données Trillium) offrant à l'élève et à ses parents une évaluation trimestrielle de la performance cumulée des tâches d'apprentissage. Les résultats au Test provincial et ceux des bulletins sont des scores de 1 à 4 de niveaux de lecture. Le Test provincial est passé une fois aux élèves à la fin de leur troisième année d'études. Les résultats en lecture issus de la FOI sont des scores de 1 à 30 qui indiquent des niveaux de lecture. Cette fiche peut être utilisée plusieurs fois durant un même trimestre ou au cours d'une année scolaire; la dernière observation de chaque trimestre est retenue comme score de progression.

\section{Les dispositifs pédagogiques mis en place}

Les nouveaux dispositifs en enseignement de la littératie instaurés dans les classes réfèrent, d'une part, à l'observation des élèves lors d'une lecture à haute voix et à l'analyse des données d'apprentissage réalisée en équipe collaborative. Ces activités ont pour but de cibler des pistes d'interventions différenciées afin de répondre aux besoins spécifiques de chaque élève. D'autre part, les nouvelles pratiques renvoient aux dispositifs documentés dans le programme équilibré d'enseignement de la lecture (Table ronde des experts, 2003, 2005; MEO, 2003). Plus précisément, lors des activités de régulation en équipe collaborative, les enseignants ont réfléchi sur ce qu'est un programme équilibré d'enseignement de la lecture (Giasson, 2011; MEO, 2003) et comment intégrer dans leur enseignement les pratiques efficaces en lecture. Ces enseignants ont porté une attention particulière aux divers éléments du programme équilibré que sont : a) l'environnement et la gestion de la classe; b) l'implantation des situations de lecture (autonome, partagée, guidée et lecture aux élèves); c) l'utilisation des données d'observation en lecture pour le regroupement des élèves lors des activités autonomes ou de lecture guidée; et d)

\footnotetext{
${ }^{6}$ Un conseil scolaire est une organisation administrative d'une région géographique donnée du système scolaire d'une province ou d'un territoire qui assume une responsabilité de soutien et de supervision éducative et financière.
} 
l'horaire quotidien équilibré d'activités en communication orale, en lecture et en écriture des diverses matières.

Pour soutenir la démarche d'implantation de nouvelles pratiques, le personnel enseignant a bénéficié d'un appui à la formation continue qui se résume en trois axes. Le premier axe concerne des sessions de formation et d'accompagnement sur l'amélioration des pratiques d'enseignement en littératie. Ces activités avaient pour but d'accompagner les enseignants dans la transposition des résultats de la recherche sur les meilleures pratiques d'enseignement en interventions efficaces en classe (programme équilibré). Le deuxième axe offre au personnel un appui à l'utilisation de grilles d'observation, mais aussi, il s'agit du (troisième axe, à l'interprétation des données d'apprentissage et à la régulation des interventions expérimentées lors des rencontres collaboratives (CAP).

\section{Les résultats}

Les premières sections décrivent les résultats quant aux apprentissages en lecture et en écriture issus des trois outils de collecte de données. Avant de décrire la progression d'apprentissage des élèves, des analyses ont permis de préciser la corrélation entre les outils d'observation ainsi que les corrélations entre les rendements en lecture et en écriture.

D'une part, l'analyse corrélationnelle rend compte d'une forte validité de convergence entre les trois mesures en lecture (résultats des FOI, résultats des bulletins et scores du Test provincial), ce qui signifie que les résultats obtenus avec chacune des trois mesures évaluent le même phénomène. Cette information justifie le choix de décrire les données d'apprentissage à partir d'un seul outil de collecte de données, soit celles issues de la FOI.

D'autre part, l'analyse corrélationnelle entre les résultats en lecture et en écriture a permis de préciser la relation entre les données d'apprentissage en lecture et ceux en écriture (années 2007 à 2010). Cette analyse fait ressortir des relations élevées entre les résultats en lecture aux trois mesures et ceux obtenus au Test provincial en écriture. Ces relations tendent à confirmer la prémisse selon laquelle il existe des processus communs d'apprentissage de la lecture et de l'écriture (Clay, 2005; Moreau \& Leclerc, 2009; Saint-Laurent, 2008). Ainsi, considérant le lien très fort entre le développement de ces deux compétences, cette information, en plus de permettre la simplification des analyses présentées, justifie le choix de ne présenter, dans cet article, que les données d'apprentissage en lecture. Les sections suivantes présentent la progression de cet apprentissage.

\section{Les trajectoires d'apprentissage en lecture}

Le croisement des résultats au Test provincial normalisé et des scores à la FOI (résultat de 1 à 30) ainsi que les analyses des scores en lecture du bulletin d'une année scolaire à une autre ont permis de décrire la nature de la progression des apprentissages en fonction des différents groupes d'élèves : ceux qui réussissent et ceux qui sont à risque. Les sections suivantes décrivent ces progressions et la nature de la trajectoire de ces apprentissages. 
La progression des apprentissages en lecture de tous les élèves. Pour tous les élèves de $3^{\mathrm{e}}$ année des huit écoles participantes (2007 à 2010), le score moyen en lecture issu de la FOI est de 25,16 $(\mathrm{n}=517)$. L'année précédente, c'est-à-dire en $2^{\mathrm{e}}$ année, ces mêmes élèves obtenaient un score moyen en lecture de 19,52 ( $\mathrm{n}=479)$. Enfin, en $1^{\text {re }}$ année, le score moyen en lecture de ces élèves était de $12,46(\mathrm{n}=265)$. En somme, la progression du niveau de lecture (FOI) correspond à un score moyen de 12,46 pour les élèves de $1^{\text {re }}$ année, de 19,52 pour ceux de $2^{\mathrm{e}}$ et de 25,12 pour ceux de $3^{\mathrm{e}}$ année.

Également, grâce à la méthodologie de courbe ROC (Receiver Operating Characteristic), cette analyse a permis de déterminer le seuil critique du niveau de lecture de la FOI qui permettra de détecter les élèves à risque (score inférieur à $3 \mathrm{au}$ Test provincial). Les caractéristiques considérées sont la sensibilité, la spécificité ainsi que l'aire sous la courbe. La sensibilité d'un test est sa capacité de détecter de vrais positifs chez les élèves plus à risque, alors que la spécificité est la probabilité d'obtenir de vrais négatifs chez les élèves moins à risque. La méthode de Youden a été retenue pour déterminer le seuil critique optimisant la sensibilité et la spécificité pour chacune des années scolaires. Ce seuil correspond à un score de 23 de la FOI pour les élèves de fin $3^{\mathrm{e}}$ année; il s'agit d'une zone de passage menant à la réussite au Test provincial.

En bref, ce score de 23 de la FOI s'inscrit dans une trajectoire de la progression du niveau de lecture des élèves qui réussissent, soit un score moyen de 12,5 en $1^{\text {re }}$ année, de 19,5 en $2^{\mathrm{e}}$ année et de 25,2 en $3^{\mathrm{e}}$ année. En outre, à la fin de la troisième année, les élèves qui sont dans la zone de passage, soit près du score 23 de la FOI, ou supérieure à celle-ci sont à un niveau de lecture qui leur permet de lire des textes sans illustration ou de construire le sens du texte sans avoir besoin de se référer à des images ou de trouver des indices de sens à l'intérieur du texte.

La progression des apprentissages en lecture des élèves à risque. La comparaison entre les résultats des trois mesures permet de distinguer les élèves qui sont à risque de ceux qui réussissent. Par cette analyse ex post facto, il a été déterminé que les élèves à risque sont ceux qui obtiennent un score inférieur au seuil de réussite (scores $<3$ ) au Test provincial (outil normalisé). L'analyse descriptive des scores en lecture (FOI) de ces élèves qui n'ont pas obtenu un score de réussite au Test provincial fournit la trajectoire de ces derniers. La progression des scores moyens en lecture (FOI) de ces élèves est de 8,7 en $1^{\text {re }}$ année, de 15,8 en $2^{\mathrm{e}}$ année et de 19,6 en $3^{\mathrm{e}}$ année.

\section{L'apport des nouveaux dispositifs d'enseignement sur les apprentissages en lecture}

L'analyse multivariée (GML) confirme que les scores en lecture provenant de la FOI (Pillai's Trace $=0,843, p<0,0001$ ) ont évolué de façon significative d'une année scolaire à l'autre (entre la $1^{\text {re }}$ et la $2^{\mathrm{e}}$ année, ainsi qu'entre la $2^{\mathrm{e}}$ et la $3^{\mathrm{e}}$ année). Cette progression est semblable pour les trois années scolaires, et ce, quel que soit le groupe auquel les élèves appartiennent : élèves qui réussissent et ceux qui sont à risque. Cette progression est associée à l'implantation de nouveaux dispositifs pédagogiques en enseignement de la littératie. La figure 1 illustre la progression des compétences en lecture des trajectoires d'élèves, ceux qui réussissent et ceux qui sont à risque, dont ceux qui ont un plan d'intervention. 


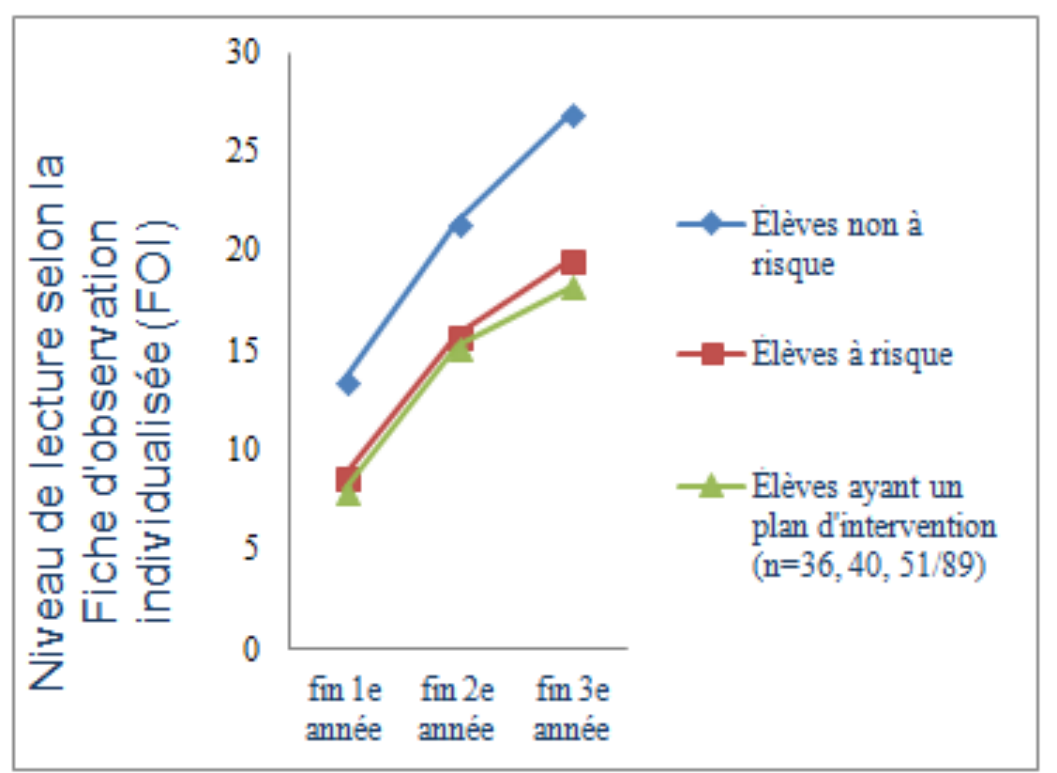

Figure 1. Scores moyens de niveaux de lecture (0-30) de la FOI, fin de la $1^{\text {re }}, 2^{\mathrm{e}}$ et $3^{\mathrm{e}}$ année d'élèves qui réussissent et ceux à risque, dont ceux ayant un plan d'intervention.

Aucune différence significative n'est observée entre les trois groupes quant à la progression des scores en lecture obtenus par la FOI. En somme, les élèves qui réussissent et ceux à risque progressent de la même façon d'une année scolaire à l'autre. De plus, bien que ces trajectoires montrent la même progression, les écarts entre les niveaux de lecture restent significativement semblables entre les deux groupes d'une année à l'autre.

\section{La synthèse}

Les analyses des mesures répétées sur trois ans du rendement en littératie (lecture-écriture) de ces élèves des huit écoles participantes font ressortir plusieurs constats.

Il existe une forte validité de convergence entre les trois mesures de l'apprentissage en lecture (Test provincial, FOI et résultats au bulletin), ce qui suggère que ces trois mesures évaluent le même phénomène.

L'analyse montre une relation entre les résultats en lecture et ceux en écriture au Test provincial, ce qui appuie la prémisse qu'il y a des processus communs dans les apprentissages de la lecture et de l'écriture.

En somme, on observe un plus grand nombre d'élèves à leur troisième année de scolarité qui obtiennent ou dépassent le seuil de réussite (score 3 ou 4) au Test provincial, ce qui tend à appuyer le lien positif entre la réussite dans les apprentissages en lecture (plus d'élèves atteignent le seuil de réussite) et les dispositifs pédagogiques centrés sur la régulation de l'enseignement, les nouvelles pratiques d'enseignement en littératie et le fonctionnement en CAP.

Pour ces élèves ayant participé à la recherche, la trajectoire de la progression du niveau de lecture (issu de la FOI) est de 9,2 en $1^{\text {re }}$ année, de 18,24 en $2^{\mathrm{e}}$ année et de 24,71 en $3^{\mathrm{e}}$ année. Une zone de passage se situe entre 19 et 26 de niveau de lecture et d'une probabilité de seuil de réussite à 23 (niveau de lecture) en $3^{\mathrm{e}}$ année. Cette trajectoire aide le personnel enseignant à intervenir de façon ciblée auprès d'élèves à risque ou de ceux qui sont près ou dans la zone de passage en s'appuyant sur une 
analyse fine des stratégies et des comportements de ces lecteurs (données d'apprentissage) et sur la régulation des interventions.

Étonnamment, une progression significative du niveau de lecture d'une année scolaire à une autre, tant pour les élèves qui réussissent que pour ceux qui sont à risque, est observée après trois ans de recherche. Selon le temps écoulé, ce résultat positif s'explique par les interventions mises en place concernant les dispositifs d'observation et de régulation des interventions associées au fonctionnement en CAP. En outre, ces résultats montrent que les interventions ciblées limitent les effets négatifs des facteurs comme le milieu socio-économique des familles sur l'apprentissage (OQRE, 2010). Somme toute, les mesures mises en place ont contré les effets liés au contexte francophone en milieu minoritaire.

Enfin, l'écart entre les deux groupes, élèves qui réussissent et ceux qui sont à risque, se maintient, ce qui confirme la prémisse que les élèves à risque bénéficient autant des interventions mises en place, et ce, sans observer l'accroissement de l'écart de niveaux de lecture entre les deux groupes. Cette équité dans les apprentissages entre élèves à risque et ceux qui réussissent constitue une donnée nouvelle dans le domaine.

\section{La discussion}

Sans vouloir nier l'influence des facteurs personnels tels que l'histoire d'apprentissage des élèves et ceux liés aux environnements familiaux et socioéconomiques (Lonigan \& Shanahan, 2010; Shanahan \& Lonigan, 2010), ces résultats montrent que les nouvelles pratiques vont dans le sens des retombées visées. Dans ces écoles fonctionnant en CAP, il s'est développé progressivement une culture de collecte et d'analyse de données d'apprentissage. La formation et l'accompagnement ont permis aux enseignants de savoir comment utiliser la FOI et d'identifier le niveau de lecture des élèves pour faire les regroupements selon des besoins ciblés et pour réaliser des activités de lecture guidée. Ces résultats vont dans le sens des recherches sur les pratiques d'enseignement en lecture auprès des élèves à risque (Allor, Champlin, Gifford, \& Mathes, 2010).

L'implantation de ces nouvelles pratiques d'enseignement passe par un solide programme de soutien à la formation fourni par le Conseil scolaire; ce qu'appuient les conclusions formulées par plusieurs chercheurs qui tendent à dire que l'amélioration des apprentissages des élèves, dont ceux ayant des difficultés, passe par un soutien à : a) l'implantation du fonctionnement en CAP (Hord \& Sommers, 2007; Moreau et al, 2013); b) l'intégration des modalités de formation continue des enseignants (SavoieZajc, 2010); et c) la mise en œuvre de nouvelles pratiques efficaces d'enseignement (Giasson, 2011; National Early Literacy Panel, 2008).

Dans le cadre de cette recherche, plusieurs enseignants ont été en mesure d'identifier les stratégies d'apprentissage et les comportements qui n'ont pas été maîtrisés par leurs élèves, d'intervenir tôt auprès des élèves à risque, dont ceux ayant un plan d'intervention, et ce, dans le cadre d'une démarche de régulation de leurs interventions (Clay, 2003). Ils sont également plus stratégiques dans leur enseignement grâce à l'analyse des données : ils choisissent un défi spécifique et essaient de le clarifier et de le surmonter avant de passer à un autre (Table ronde des experts, 2005, 2003). Ces changements de pratiques expliquent, en partie, l'amélioration des résultats d'apprentissage en lecture chez les élèves au cours de ces trois années de recherche.

Somme toute, ces résultats s'apparentent à la conclusion de Bélanger, Bowen, Cartier, Desbiens, Montésinos-Gelet \& Turcotte (2012), c'est-à-dire que vue comme 
un processus complexe et dynamique, l'implantation de nouvelles pratiques efficaces, pour qu'elles puissent être effectives à long terme, exige des mesures de régulation et d'ajustement constants en plus de conditions favorables à leur mise en œuvre. Ces conditions sont remplies lorsqu'il y a un équilibre entre la capacité à documenter et à analyser les données d'apprentissage des élèves, la capacité à intégrer dans la pratique en enseignement les façons de faire qui répondent aux besoins des élèves, dont les innovations issues des résultats probants de recherches et, enfin, la capacité à fonctionner en CAP (Leclerc \& Moreau, 2010). C'est par un fonctionnement scolaire de collaboration favorisant la pratique réflexive entre enseignants, un modelage à travers les observations entre collègues ainsi qu'une rétroaction de ces derniers qu'il est possible de prétendre à une mise en œuvre de pratiques innovantes. Ces moyens s'avèrent essentiels pour observer des changements (Bélanger et al, 2012).

Pour les élèves à risque, la mise en place de modalités renouvelées d'enseignement en littératie leur permet d'apprendre à lire et de progresser dans leurs apprentissages comme tout autre élève. Sans rattraper leur retard, la progression de leurs apprentissages décrite dans la présente recherche est significative d'une année scolaire à l'autre. Ces résultats suggèrent que la qualité des enseignements explicites ainsi que la précocité, la spécificité et l'intensité de l'intervention, à savoir un dépistage précoce des difficultés en apprentissage de la littératie, une précision des besoins des élèves jumelée à une intervention en petits groupes et des niveaux de textes qui correspondent à leurs besoins, sont quelques ingrédients incontournables à la réussite de l'apprentissage (Vaughn et al., 2010; Wanzek \& Vaughn, 2007).

\section{Conclusion}

Cette recherche longitudinale a permis de décrire la progression des apprentissages de la lecture selon différentes caractéristiques d'élèves en milieu minoritaire, soit ceux qui réussissent et ceux qui sont à risque. Ces descriptions et les analyses de la progression des apprentissages ont permis de répondre aux questions visant à décrire les trajectoires d'apprentissage d'élèves et à préciser l'apport de l'implantation de nouveaux dispositifs d'enseignement en littératie sur les compétences en littératie (lecture-écriture). Sans pouvoir énoncer de conclusions généralisables, ces résultats fournissent des tendances: a) les élèves à risque bénéficient de l'implantation de nouvelles pratiques efficaces d'enseignement différencié en littératie; et b) les dispositifs d'enseignement favorisent le développement des compétences en littératie et réduisent les écarts entre les élèves, peu importe leurs caractéristiques (forts et faibles) (Bressoux, 2001). Ces nouvelles pratiques s'appuient sur diverses modalités que sont le fonctionnement en CAP, le dépistage précoce des difficultés, les différentes modalités liées à l'enseignement explicite et un niveau approprié de l'intensité de l'intervention.

Dans le cadre de cette recherche, la concertation-coordination et l'arrimage entre l'implantation du fonctionnement en CAP jumelé à la formation, l'appropriation de nouvelles pratiques d'observation des apprentissages (FOI) et les changements de pratiques en enseignement en littératie ont été possibles grâce à des orientations claires et à l'engagement des enseignants et des membres des directions d'écoles et du Conseil scolaire à la formation-soutien. Ces facteurs structurels sont déterminants. Également, ajoutons à ces facteurs la croyance chez ces membres de l'importance des activités de recherche comme outil à l'amélioration des pratiques. Ces facteurs caractérisent cette recherche.

En outre, l'enseignement des compétences en littératie en contexte d'inclusion et en milieu minoritaire est complexe et exige la mise en place de plusieurs 
interventions hors de l'école, dans l'école et dans les classes. Inspirée de l'approche préventive des difficultés d'apprentissage, cette recherche décrit certains dispositifs pédagogiques impliquant l'articulation de ces niveaux d'intervention, ce qui rend possible des changements dans l'école et dans les classes. Toutefois, dans cette recherche, les différents dispositifs et éléments privilégiés par les enseignants empêchent de préciser la contribution singulière de chacun de ceux-ci sur les apprentissages. Donc, il faut être prudent dans l'interprétation de ces résultats; il s'agit de l'une des limites de cette recherche.

Les recherches futures doivent permettre de préciser l'influence de ces différents facteurs que sont le fonctionnement en CAP et l'intensité des interventions en petits groupes. Dans le même sens, les recherches futures doivent documenter les modalités d'appropriation de ces dispositifs par le personnel enseignant au regard de la formation continue, entre autres, l'apport de nouvelles technologies. Enfin, de nouvelles recherches doivent étudier le développement du langage oral et écrit auprès d'élèves en milieu minoritaire francophone.

\section{Références}

Allor, J. H., Champlin, T. M., Gifford, D. B., \& Mathes, P. G. (2010). Méthodes pour augmenter l'intensité de l'enseignement de la lecture pour les élèves ayant une déficience intellectuelle. L'éducation et la formation en autisme et déficience développementale, 45(4), 500-511.

Allor, J. H., Mathes, P. G., Roberts, J. K., Cheatham, J. P., \& Champlin, T. M. (2010). Enseignement de la lecture complète pour les étudiants ayant une déficience intellectuelle: Les résultats des trois premières années d'une étude longitudinale. La psychologie dans les écoles, 47, 445-466.

Bara, F., Gentaz, É., \& Colé, P. (2004).Les effets des entraînements phonologiques et multisensoriels destinés à favoriser l'apprentissage de la lecture chez les jeunes enfants. Enfance, 56(4), 387-403.

Baylis, P., \& Snowling, M. (2012). Évaluation d'un programme de lecture phonologique pour les enfants atteints du syndrome de Down. L'enseignement des langues des enfants et la thérapie, 28(1), 39-5.

Bélanger, J., Bowen, F., Cartier, S., Desbiens, N., Montésinos-Gelet, I. et Turcotte, L. (2012). L'appropriation de nouvelles pratiques d'interventions pédagogiques et éducatives en milieu scolaire: Réflexions sur un cadre théorique intégrateur. Éducation et francophonie, 40(1), 56-75.

Blanc, N. (2009). Lecture et habiletés de compréhension chez l'enfant. Paris, France : Dunod.

Blanc, N., \& Brouillet, D. (2003). Mémoire et compréhension : lire pour comprendre. Paris, France : Éditions In press.

Bressoux, P. (2001). Réflexions sur l'effet-maître et l'étude des pratiques enseignantes. Les dossiers des sciences de l'éducation. 5, 9-35.

Browder, D. M., Ahlgrim-Delzell, L., Courtade, G., Gibbs, S. L., \& Flowers, C. (2008). Evaluation of the effectiveness of an early literacy program for students with significant developmental disabilities using group randomized trial research. Exceptional Children, 75, 33-52.

Burns, M. K., Appleton, J. J., \& Stehouwer, J. D. (2005). Meta-analytic review of responsiveness-to-intervention research: Examining field-based and researchimplemented models. Journal of Psychoeducational Assessment, 23(4), 381394. 
Clay, M. M. (2003). Le sondage d'observation en lecture-écriture. Montréal, Québec: Chenelière/McGraw-Hill.

Clay, M. M. (2005). Literacy lessons: Designed for individuals, Part one: Why? When? and How? Birkenhead, Auckland, Nouvelle-Zélande : Heinemann.

Conseil canadien sur l'apprentissage. (2011). Quel est le futur de l'apprentissage au Canada? Ottawa, Ontario: Conseil canadien sur l'apprentissage. Repéré à http://www.ccl-cca.ca/pdfs/CEOCorner/2010-10-

11QuelestlefuturdelapprentissageauCanada.pdf

Conseil canadien sur l'apprentissage. (2007). État de l'apprentissage au Canada: Pas le temps de s'illusionner, Rapport sur l'apprentissage au Canada. Ottawa, Ontario : Conseil canadien sur l'apprentissage.

Conseil supérieur de l'éducation. (2010). Rapport sur l'état et les besoins de l'éducation 2008-2010: Conjuguer équité et performance en éducation, un défi de société. Québec, Québec: Gouvernement du Québec. Repéré à http://www.cse.gouv.qc.ca/fichiers/documents/publications/CEBE/500192.pdf

Cormier, P. (2006). Connaissance du nom des lettres chez des enfants francophones de 4, 5 et 6 ans au Nouveau-Brunswick. Éducation et francophonie, 34(2), 527.

Courcy, A. (2000). Conscience phonologique et apprentissage de la lecture. Montréal, Québec : Université de Montréal.

Crahay, M. (2000). L'école peut-elle être juste et efficace? De l'égalité des chances à l'égalité des acquis. Bruxelles, Belgique : De Boeck et Larcier s.a.

Dufour, R., \& Eaker, R. (2004). Communautés d'apprentissage professionnelles: Méthodes d'amélioration du rendement scolaire. Bloomington, IN : National Education Service.

Eaker, R., Dufour, R., \& Dufour, R. (2004). Premiers pas : Transformation culturelle de l'école en communauté d'apprentissage professionnelle. Bloomington, IN : National Educational Service.

Ecalle, J., \& Magnan, A. (2010). L'apprentissage de la lecture et ses difficultés. Paris, France : Armand Colin Éditeur.

Ecalle, J., \& Magnan, A. (2002). L'apprentissage de la lecture : Fonctionnement et développement cognitif. Paris, France : Armand Colin Éditeur.

Fitzgerald, J., \& Shanahan, T. (2000). Reading and Writing Relations and Their Development. Educational Psychologist, 35 (1), 39-50.

Foulin, J. N. (2007). La connaissance des lettres chez les prélecteurs : aspects pronostiques, fonctionnels et diagnostiques. Psychologie française, 112, 1-14.

Fountas, I., \& Pinnell, G. S. (2001). Guiding readers and writers grades 3-6; Teaching comprehension, genre and content literacy. Portsmouth, NH: Heinemann.

Fullan, M. (2001). The New Meaning of Educational Change ( $3^{\text {rd }}$ Ed). NewYork, NY : Teachers College Press.

Giasson, J. (2011). La lecture: apprentissage et difficultés. Montréal, Québec: Gaétan Morin.

Grenier, S., Jonse, S., Strucker, J., Murray, T. S., Gervais, G., \& Brink, S. (2008). Enquête internationale sur l'alphabétisation des adultes. L'apprentissage de la littératie au Canada: Constatations tirées de l'Enquête internationale sur les compétences en lecture. Ottawa, Ontario : Statistique Canada, Ressources humaines et Développement social Canada. 
Hord, S. M., \& Sommers, W. (2007). Leading Professional Learning Communities: Voices from Research and Practice. Thousand Oaks, CA: Corwin Press.

Jaffré, J.-P. (2003). La linguistique et la lecture-écriture : de la conscience phonologique à la variation «orthographe». Revue des sciences de l'éducation, 29, 37-49.

Landry, R. (2003). Libérer le potentiel caché de l'exogamie. Profil démolinguistique des enfants des ayant droits francophones selon la structure familiale. Moncton, Nouveau-Brunswick: Institut canadien de recherches sur les minorités linguistiques, Université de Moncton.

Leclerc, M., \& Moreau, A. C. (2010). La diffusion de l'expertise au sein de la communauté d'apprentissage professionnelle comme levier de réussite des élèves. Le Point en administration de l'éducation, 12(4), 22-28.

Leclerc, M., \& Moreau, A. C. (2009). Toujours plus loin en littératie : Rapport de recherche. Gatineau, Québec : GRECIP, Université du Québec en Outaouais.

Legendre, R. (2005). Dictionnaire actuel de l'éducation. Montréal, Québec : Guérin.

Lemons, C. J., \& Fuchs, D. (2010). Phonological awareness of children with Down syndrome: Its role in learning to read and the effectiveness of related interventions. Research in Developmental Disabilities, 31(2), 316-330.

Lonigan, C. J., \& Shanahan, T. (2010). Developing Early Literacy Skills: Things W Know We Know and things We Know We Don't Know. Educational Researcher: An Official Journal of the American Educational Research Association, Special issue, 19(4), 340-346.

Makdissi, H., \& Boisclair, A. (2006). Modèle d'intervention pour l'émergence de la littératie. Nouveaux cahiers de la recherche en éducation, 9(2), 147-169.

Mallozzi, C. A., \& Malloy, J. A. (2007). Reading and writing connections. Reading Research Quarterly, Reports on Literacy Research. Reading Research Quarterly, 42(3), 161-166.

McCardle, P., Chhabra, V., \& Kapinus, B. (2008). Reading Research in Action: A Teacher's Guide for Student Success. Baltimore, MD : Brookes Publishing Co.

Miffre, M. (1992). Apprentissage de la régulation pédagogique en formation professionnelle des maîtres. SPIRALE - Revue de Recherches en Éducation, 8, 87-95.

Ministère de l'Éducation de l'Ontario (MÉO) (2003). Guide d'enseignement efficace de la lecture de la maternelle à la $3^{e}$ année. Toronto, Ontario : ministère de l'Éducation de l'Ontario.

Ministère de l'Éducation du Québec (MEQ) (2001). Programme de formation à l'enseignement. Repéré à

http://www.mels.gouv.qc.ca/references/publications/resultats-de-larecherche/detail/article/la-formation-a-lenseignement-les-orientations-lescompetences-professionnelles/

Montésinos-Gelet, I. (2009). La démarche des orthographes approchées en contexte francophone minoritaire. Dans D. Masny (dir.), Lire le monde. Les littératies multiples et l'éducation dans les communautés francophones (p. 21-31). Ottawa, Ontario : Les Presses de l'Université d'Ottawa.

Moreau, A.C., Hébert, M., Lépine, M., \& Ruel, J. (2013). Le concept de littératie en francophonie: que disent les définitions? Revue Consortium national de recherche sur l'intégration sociale (CNRIS), 4(2), 14-18.

Moreau, A., C., \& Leclerc, M. (2009). Recherche de sens chez les enseignants au regard des relations entre l'oral, la lecture et l'écriture. Revue de l'Association belge pour la lecture (ABLF) Caractères, 33, 34-40. 
Moreau, A.C., Leclerc, M., \& Stanké, B. (2013). L'apport du fonctionnement en communauté d'apprentissage professionnelle de huit écoles sur l'autoappréciation en enseignement en littératie et sur le sentiment d'autoefficacité. Éducation et francophonie, XLI(2), 35-61.

National Early Literacy Panel. (2008). Developing early literacy. A scientific synthesis of early literacy development and implications for intervention. Repéré à http://lincs.ed.gov/publications/pdf/NELPReport09.pdf

National Reading Panel. (2000). Teaching children to read: an evidence-based assessment of the scientific research literature on reading and its implications for reading instruction: Reports of the subgroups. Washington, DC: Government Printing Office.

Nelley, E., \& Smith, A. (2003). Trousse d'évaluation en lecture GB+. Laval, Québec : Groupe Beauchemin.

Office de la qualité de la responsabilité en éducation (OQRE). (2010). Programme international pour le suivi des acquis des élèves (PISA), 2009: Faits saillants des résultats des élèves de l'Ontario. Toronto, Ontario: OQRE. Repéré à http://www.eqao.com/pdf f/10/2009_PISA_highlights_fr.pdf

Office de la qualité de la responsabilité en éducation (OQRE). (2009). Rapport $d u$ conseil scolaire CSDC Centre-Sud (67318). Toronto, Ontario : OQRE. Repéré à

http://eqaoweb.eqao.com/eqaoweborgprofile/profile.aspx?Lang=F\& Mident= 727270

Perrenoud, P. (1998). L'évaluation des élèves. De la fabrication de l'excellence à la régulation des apprentissages. Paris, France et Bruxelles, Belgique : De Boeck et Larcier s.a.

Reuter, Y. (1998). Les interactions lecture-écriture. Actes du colloque Théodile-Crel (Lille, novembre 1993). Bern, Suisse : Peter Lang S.A.

Ridgeway, T. R., Price, D. P., Simpson, C. G., \& Rose, C. A. (2012). Reviewing the roots of response to intervention: Is there enough research to support the promise? Administrative Issues Journal: Education, Practice \& Research, 2(1), 83-95.

Rousseau, N. (2010). La pédagogie de l'inclusion scolaire: Piste d'action pour apprendre tous ensemble ( $2^{\mathrm{e}}$ éd.). Sainte-Foy, Québec : Presses de 1'Université du Québec

Saint-Laurent, L. (2008). Enseigner aux élèves à risque et en difficulté au primaire. Montréal, Québec : Chenelière Éducation.

Savoie-Zajc, L. (2010). Les dynamiques d'accompagnement dans la mise en place de communautés d'apprentissage de personnels scolaires. Revue Éducation \& Formation, 292, 9-20.

Scheffel, D. L., Shaw, J. C., \& Shaw, R. (2008). The efficacy of a supplemental multisensory reading program for first grade students. Reading Improvement, $45(3), 139-152$.

Secrétariat de la littératie et de la numératie (2007). Cadre pour l'efficacité des écoles: un processus collectif d'amélioration continue de l'efficacité des écoles élémentaires de l'Ontario. Toronto, Ontario : ministère de l'Éducation de l'Ontario.

Shanahan, T., \& Lonigan, C. J. (2010). The National Early Literacy Panel: A Summary of the Process and the Report. Educational Researcher: An Official Journal of the American Educational Research Association, Special issue, 19(4), 279-283. 
Table ronde des experts. (2003). Stratégie de lecture au primaire: Rapport de la Table ronde des experts en lecture. Toronto, Ontario : ministère de l'Éducation de l'Ontario.

Table ronde des experts. (2005). L'éducation pour tous : Rapport de la Table ronde des experts pour l'enseignement en matière de littératie et de numératie pour les élèves ayant des besoins particuliers de la maternelle à la $\sigma^{e}$ année. Toronto, Ontario : ministère de l'Éducation de l'Ontario.

Van den Broek, P.W., Kendou, P., Kremer, K., Lynch, J.S., Butler, J., White, M.J., \& Lorch, E.P. (2005). Assessment of comprehension abilities in young children. Dans S. Stahl et S. Paris (dir.), Children's Reading Comprehension and Assessment. Center for the Improvement of Early Reading Achievement ( $\mathrm{p}$. 107-130). Mahwah, NJ : Lawrence Earlbaum Associates.

Vaughn, S., Denton, C. A., \& Fletcher, J. M. (2010). Why intensive interventions are necessary for students with severe reading difficulties. Psychology Science, 47(5), 432-444. Repéré à http://www.ncbi.nlm.nih.gov/pmc/articles/PMC2975106/

Wanzek, J., \& Vaughn, S. (2007). Research-based implications from extensive early reading interventions. School Psychology Review, 36, 541-561.

\section{Les biographies des auteurs}

André C. Moreau, professeur chercheur à l'Université du Québec en Outaouais réalise des recherches avec les réseaux de l'éducation de la santé et des services sociaux comme orthopédagogue-psychopédagogue. Ses recherches portent sur l'inclusion des élèves ayant des besoins particuliers en milieux scolaires et sur les pratiques d'enseignement-apprentissage des compétences en littératie. Des recherches-actions et en collaboration ont également été réalisées sur les thèmes des transitions scolaires et de l'implantation du fonctionnement d'écoles inclusives en communauté d'apprentissage professionnelle. Membre d'Équipes de recherche en littératie et inclusion (ÉRLI), il est cotitulaire de la Chaire interdisciplinaire de littératie et inclusion - Pavillon du Parc (CIRLI).

Brigitte Stanké est professeure à l'université de Montréal. Elle détient une maîtrise et un doctorat en orthophonie et audiologie à l'université de Montréal ainsi qu'une maîtrise en mathématiques à l'université de Montréal. Orthophoniste, elle a travaillé plus de 15 ans en milieu scolaire auprès d'enfants et d'adolescents présentant des difficultés de langage oral et écrit. Ses recherches portent principalement sur l'apprentissage de la lecture et de l'orthographe, de ses difficultés et de sa remédiation. Auteure de plusieurs ouvrages à succès visant à développer le langage oral et écrit, elle est reconnue au Québec, au Canada et en Europe ou elle anime régulièrement des sessions de formation portant sur le dépistage des troubles d'apprentissage, la dyslexie-dysorthographie et la dyscalculie.

Joanne Pharand est professeure agrégée en formation pratique à l'Université du Québec en Outaouais, campus de Saint-Jérôme, où elle est responsable pédagogique des stages au baccalauréat en éducation préscolaire et enseignement primaire et responsable de la formation des enseignants associés. Elle est membre d'ÉRLI (Équipe de recherche en littératie et inclusion) et s'intéresse particulièrement à la littératie émotionnelle au primaire en enseignement/apprentissage. Ses recherches 
portent aussi sur les compétences attendues des principaux acteurs impliqués dans les stages ainsi que sur l'autoformation en insertion professionnelle.

Lizanne Lafontaine est professeure titulaire de didactique du français au Département des sciences de l'éducation de l'Université du Québec en Outaouais, campus Saint-Jérôme, et responsable de l'Équipe de recherche en littératie et inclusion (ÉRLI) de la même université. Ses recherches portent sur la didactique de l'oral au préscolaire, au primaire et au secondaire ainsi que sur la littératie en milieu défavorisé. 